\title{
Effect of Environmental Hypothermia on Vitelline Artery Blood Pressure and Vascular Resistance in the Stage 18, 21, and 24 Chick Embryo
}

\author{
MAKOTO NAKAZAWA, EDWARD B. CLARK, NORMAN HU, AND JON WISPÉ \\ Division of Pediatric Cardiology, Department of Pediatrics, and The Cardiovascular Center, University of Iowa \\ Hospitals and Clinics, Iowa City, Iowa 52242 and Department of Pediatric Cardiology, The Heart Institute of \\ Japan, Tokyo Women's Medical College, Tokyo, Japan
}

\begin{abstract}
We studied the effect of environmental hypothermia on arterial blood pressure, dorsal aortic blood flow, and vascular resistance in stage 18, 21, and 24 chick embryos. The arterial pressure was measured with a servonull micropressure system. Mean dorsal aortic blood flow was calculated from pulsed-Doppler measurement of mean dorsal aortic blood velocity and dorsal aortic diameter. Vascular resistance was calculated by dividing mean vitelline arterial blood pressure by dorsal aortic blood flow. Sequential data were obtained at temperatures of 34.7, 31.1, and $34.1^{\circ} \mathrm{C}$. At stage 21, the vitelline arterial blood pressure decreased from $0.82 \pm 0.03(\overline{\mathrm{x}} \pm \mathrm{SEM})$ to 0.72 $\pm 0.03 \mathrm{~mm} \mathrm{Hg}$ on cooling and increased from $0.66 \pm 0.05$ to $0.87 \pm 0.06 \mathrm{~mm} \mathrm{Hg}$ on rewarming $(p<0.05)$. At stage 21 , mean dorsal aortic blood flow decreased from $0.49 \pm$ 0.02 to $0.33 \pm 0.02 \mathrm{~mm}^{3} / \mathrm{s}$ with cooling and increased from $0.34 \pm 0.02$ to $0.47 \pm 0.02 \mathrm{~mm}^{3} / \mathrm{s}$ with rewarming. The vascular resistance in stage 21 embryos increased after cooling from $1.68 \pm 0.19$ to $2.23 \pm 0.39 \mathrm{~mm} \mathrm{Hg} / \mathrm{mm}^{3} / \mathrm{s}(\overline{\mathbf{x}}$ $\pm 95 \%$ confidence interval). The changes were similar in stage 18 and 24 embryos. We conclude that the reduction of vitelline artery blood pressure resulted from a decrease in cardiac output. In addition, we noted that the vitelline arterial vascular bed can constrict in response to hypothermia prior to autonomic innervation. These changes in hemodynamics may be a teratogenic mechanism for hypothermia-induced cardiac defects in the chick embryo. (Pediatr Res 19:651-654, 1985)
\end{abstract}

Low environmental temperature is one of the hazards to the chick embryo during incubation. In experimental studies, a decrease in incubation temperature causes embryo death or malformations of various organs including the heart (1). Hypothermia also results in hemodynamic changes including a decrease in heart rate and dorsal aortic blood flow in the embryo (2). There are, however, no integrated studies on the effects of hypothermia on blood pressure and vascular resistance.

We studied the effect of hypothermia on vitelline artery blood pressure, dorsal aortic blood flow, and vascular resistance in the stage 18,21 , and 24 chick embryos, points in development prior to the functioning of the autonomic nervous system. We found

Received May 2, 1984; accepted February 20, 1985.

Correspondence to Edward B. Clark, M.D., Division of Pediatric Cardiology, Brady 516, Johns Hopkins Hospital, 600 N. Wolfe St. Baltimore, MD 21205.

Supported by NIH Grant HL-14388. E.B.C. is the recipient of NIH Research Career Development Award HD-00376. that blood pressure and cardial output decreased and vascular resistance increased with hypothermia in all stages.

\section{METHODS}

Fertile white Leghorn chicken eggs were incubated blunt end up in a forced draft constant humidity incubator to Hamburger and Hamilton (3) stages 18 (approximately 3 days), 21 (31/2 days), and 24 (4 days). We opened the shell and removed the outer and inner shell membranes to expose the embryo. We inserted a 5 $\mu \mathrm{m}$ diameter-tipped drawn glass micropipette electrode in a firstorder vitelline artery for measuring pressure with a servo-null micropressure system (model 900, WP Instruments). We previously showed this technique is linear over a range of $0-30 \mathrm{~mm}$ $\mathrm{Hg}\left(\mathrm{y}=0.995 \times-0.23, r^{2}=0.99, \mathrm{SEE}=0.11 \mathrm{~mm} \mathrm{Hg}\right)(4)$. Environmental temperature was monitored with a $1-\mathrm{mm}$ thermistor probe placed adjacent to the embryo. Since it was not technically possible to measure embryo body temperature directly, we assumed that environmental temperature accurately reflected embryo core temperature.

Mean dorsal aortic blood flow, an index of cardiac output, was determined by measuring mean dorsal aortic blood velocity with a $20 \mathrm{MHz}$ pulsed-Doppler velocity meter. The $1-\mathrm{mm}$ piezoelectric crystal was positioned over the dorsal aorta at a $45^{\circ}$ angle. We previously showed that this technique is linear over a range of $0-16 \mathrm{~mm} / \mathrm{s}\left(\mathrm{y}=0.85 \mathrm{x}+1.15, r^{2}=0.99, \mathrm{SEE}=0.49 \mathrm{~mm} / \mathrm{s}\right)$ (4). Dorsal aortic diameter was measured with a filar micrometer eyepiece calibrated against a $10-\mu \mathrm{m}$ scribed glass standard. Mean dorsal aortic blood flow was calculated as the product of mean blood velocity and aortic area. Vascular resistance was calculated by dividing mean vitelline artery blood pressure by dorsal aortic blood flow.

After baseline recording at a temperature of $34.7^{\circ} \mathrm{C}$, the egg was exposed to cool air for approximately $5 \mathrm{~min}$ until the environmental temperature reached $31.1^{\circ} \mathrm{C}$. We then recorded blood pressure and heart rate. The egg was then rewarmed by warm air for approximately $5 \mathrm{~min}$ to a temperature of $34.1^{\circ} \mathrm{C}$.

The experimental recordings were divided into two parts. Arterial pressure was recorded in a group of embryos during cooling and a separate group of embryos during rewarming. This was necessary to avoid alteration of the baseline when hypertonic electrolyte solution diffused within the micropipette shifting the resistance of the system.

Since duplicate individual measurements were not performed on the same embryo, mean and $95 \%$ confidence limits for vascular resistance were determined by ratio analysis of the mean and SD of pressure and flow at each stage (5). Blood pressure, mean dorsal aortic blood flow, and heart rate data are presented 
as mean \pm SEM. We used a paired $t$-test to analyze these results. Probabilities of less than $5 \%(p<0.05)$ are presented as statistically significant.

\section{RESULTS}

Mean vitelline artery blood pressure decreased significantly on cooling and returned to baseline measurements with rewarming at each stage (Fig. 1; Table 1). Mean dorsal aortic blood flow also decreased significantly with cooling and returned to baseline with rewarming (Fig. 2). Heart rate decreased on cooling but did not completely return to baseline on rewarming (Fig. 3).

Vascular resistance increased significantly with cooling at each stage studied (Fig. 4). With rewarming, the vascular resistance in stage 21 and 24 embryos showed a decreasing trend but increased slightly in stage 18 embryos.

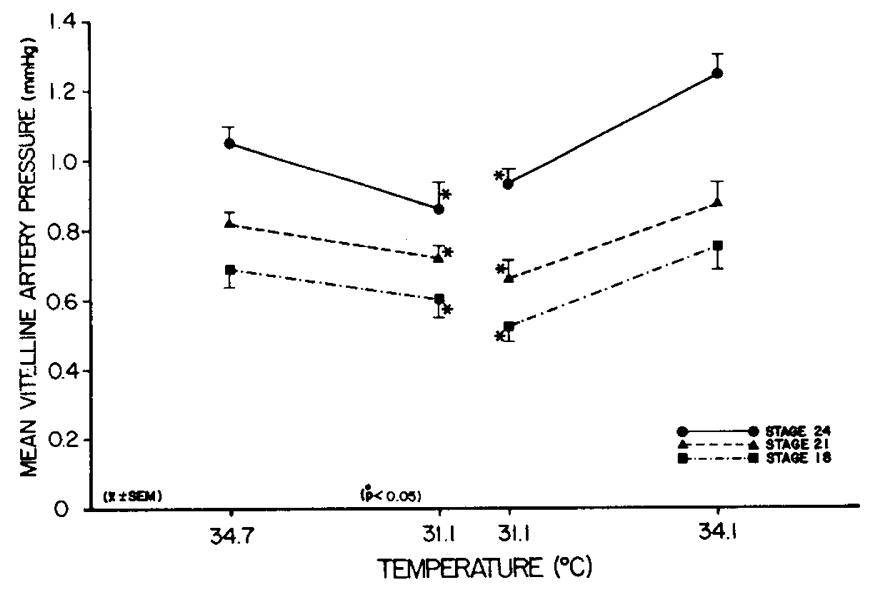

Fig. 1. Mean vitelline artery pressure versus environmental temperature for experimental embryo at stages 18,21 , and 24. Separate groups of embryos were studied for the response to cooling and rewarming (see text). Asterisks indicate statistically differences $(p<0.05)$ from initial temperature.

\section{DISCUSSION}

We are studying the functional characteristics of the chick embryo cardiovascular system. We previously described the changes in cardiac output, heart rate, and vascular resistance that occur as the embryo develops (4). As a natural extension of these studies, we sought to define the embryos response to environmental temperature changes.

Since the chick embryo is poikilothermic, it is important to determine the cardiovascular response to a decrease in environmental temperature, a naturally occurring risk during incubation. Previous investigators have shown that low environmental temperature decreases heart rate in the chick embryo (6) as well as in cultured heart cells (7). We reported that the decrease in embryo heart rate results in a decrease in dorsal aortic blood flow, an index of cardiac output. However, stroke volume remains unchanged (2). We speculated that this response is a protective mechanism to compensate for the effect of temperature fluctuation during incubation.

In order to have a comprehensive picture of the embryos response to hypothermia, it is important to know what changes occur in arterial pressure and resistance. Girard (8) showed that

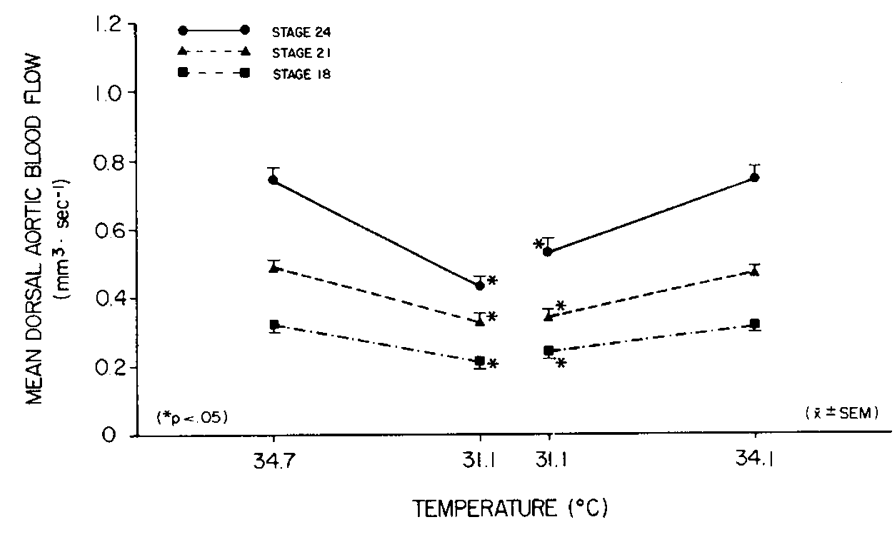

Fig. 2. Mean dorsal aortic blood flow versus environmental temperature for experimental embryos at stages 18, 21, and 24.

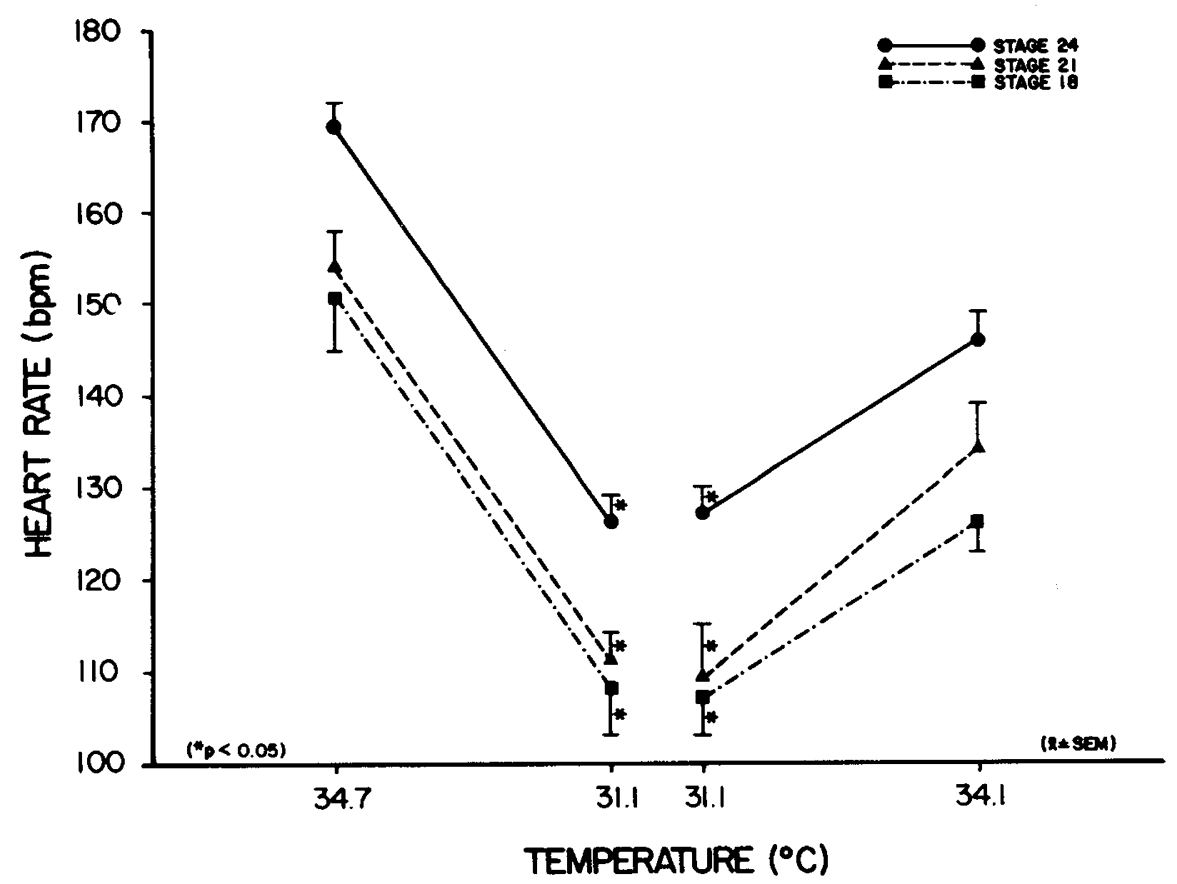

Fig. 3. The change in heart rate observed among stage 18,21 , and 24 experimental embryos following cooling from 34.7 to $31.1^{\circ} \mathrm{C}$ and after rewarming from 31.1 to $34.1^{\circ} \mathrm{C}$. 


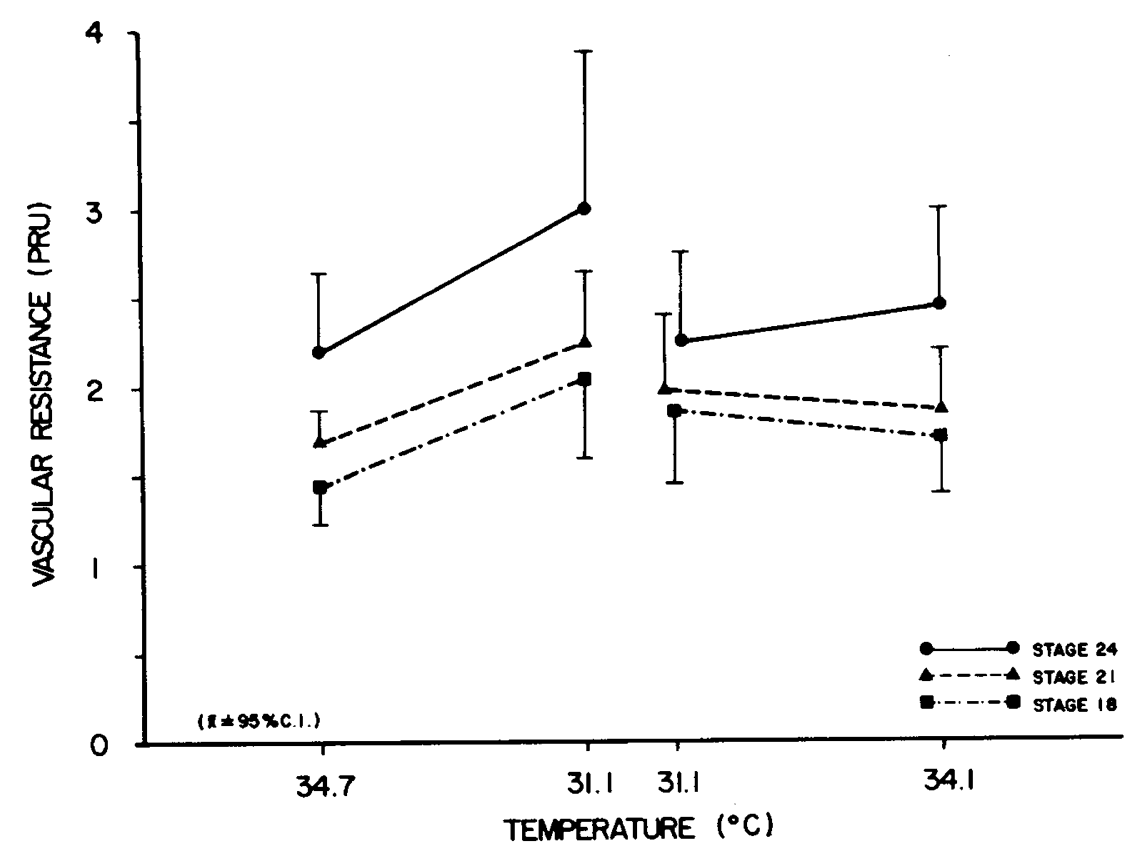

Fig. 4. Mean vascular resistance ( $\pm 95 \%$ confidence interval) versus environmental temperature for experimental embryos at stages 18,21 , and 24.

Table 1. Heart rate, mean vitelline artery pressure, mean dorsal aortic blood flow, and vascular resistance by stage at each of the environmental temperatures (mean $\pm S E M$ )

\begin{tabular}{lccccc}
\hline & Stage & $34.7^{\circ} \mathrm{C}$ & $31.1^{\circ} \mathrm{C}$ & $31.1^{\circ} \mathrm{C}$ & $34.1^{\circ} \mathrm{C}$ \\
\hline Heart rate (bpm) & 18 & $151 \pm 6$ & $108 \pm 5$ & $107 \pm 4$ & $126 \pm 3$ \\
& 21 & $154 \pm 4$ & $111 \pm 3$ & $109 \pm 6$ & $134 \pm 5$ \\
& 24 & $169 \pm 3$ & $126 \pm 3$ & $127 \pm 3$ & $146 \pm 3$ \\
Mean vitelline artery pressure (mm Hg) & & & & \\
& 18 & $0.69 \pm 0.05$ & $0.60 \pm 0.05$ & $0.52 \pm 0.04$ & $0.75 \pm 0.06$ \\
& 21 & $0.82 \pm 0.03$ & $0.72 \pm 0.03$ & $0.66 \pm 0.05$ & $0.87 \pm 0.06$ \\
Mean dorsal aortic blood flow $\left(\mathrm{mm}^{3} / \mathrm{s}\right)$ & 24 & $1.05 \pm 0.04$ & $0.86 \pm 0.07$ & $0.93 \pm 0.04$ & $1.24 \pm 0.06$ \\
& & & & \\
& 18 & $0.32 \pm 0.02$ & $0.21 \pm 0.02$ & $0.24 \pm 0.02$ & $0.31 \pm 0.01$ \\
Vascular resistance $\left(\mathrm{mm} \mathrm{Hg} / \mathrm{mm}^{3} / \mathrm{s}\right)(\overline{\mathrm{x}} \pm$ & 21 & $0.49 \pm 0.02$ & $0.33 \pm 0.02$ & $0.34 \pm 0.02$ & $0.47 \pm 0.02$ \\
95\% confidence interval) & 24 & $0.74 \pm 0.04$ & $0.43 \pm 0.03$ & $0.53 \pm 0.04$ & $0.74 \pm 0.04$ \\
& & & & \\
& 18 & $2.18 \pm 0.43$ & $3.00 \pm 0.86$ & $2.22 \pm 0.53$ & $2.44 \pm 0.53$ \\
& 21 & $1.68 \pm 0.19$ & $2.23 \pm 0.39$ & $1.98 \pm 0.42$ & $1.86 \pm 0.32$ \\
& 24 & $1.44 \pm 0.23$ & $2.04 \pm 0.45$ & $1.81 \pm 0.37$ & $1.71 \pm 0.30$ \\
\hline
\end{tabular}

arterial blood pressure decreased in both the 3- and 7-day chick embryo in response to hypothermia. Warbanow (9) demostrated a similar response in embryos at 16 days of incubation. However, in neither of these studies was cardiac output measured and consequently vascular bed resistance could not be determined.

In the current study, we measured the effect of environmental cooling on arterial blood pressure, cardiac output index, and calculated vascular resistance. We confirmed that arterial blood pressure in stage 18,21 , and 24 chick embryos decreased in response to environmental hypothermia. Our calculations indicate that the chick embryo compensates for a decrease in cardiac output by increasing vascular resistance at stages 18,21 , and 24 . The subsequent return of vascular resistance toward baseline following rewarming suggests that this response is reversible and likely a mechanism important in cardiovascular regulation of the primitive circulation.

Maintenance of blood pressure would preserve perfusion of primitive embryonic blood vessels that supply the rapidly developing organs. The persistence or disappearance of vascular channels such as the aortic arch is dependent on blood flow and pressure which maintain vessel patency (10).
We expect that the resistance is modulated in the extraembryonic vascular bed. The vascular plexus of the embryonic circulation is very primitive and devoid of smooth muscles at these stages while the extraembryonic vascular bed is more histologically complex.

In mature animals, a change in peripheral resistance is a protective mechanism. In mammals with a functional autonomic nervous system, hypothermia causes peripheral vasoconstriction through both direct action on the resistance vessels and an increase in sympathetic tone. The response to a cool environment compensates for a decrease in cardiac output (11). Our study in the chick embryo, suggests that an increase in vascular resistance is a very primitive response that compensates for environmental hypothermia prior to autonomic innervation.

At stages 18 to 24 , the autonomic nervous system is probably not functional. Functional parasympathetic and sympathetic innervation of the heart occurs at stages 38 and 41 , respectively $(12,13)$. The time of functional innervation of the extraembryonic vascular bed has not yet been defined. However, anatomic intervention of extraembryonic vasculature is derived from the prevertebral plexus which forms during the 5 th and 6 th days 
of incubation and postganglionic nerves are found in the peritoneal cavity by 10 days (14). Therefore, it is unlikely that the extraembryonic vascular is innervated during the period we studied.

The chick embryo's vascular bed is dynamic. Although the adrenergic nervous system is not functional, adrenergic receptors are present in the heart and vascular system. This is not surprising since adrenergic-like receptors are phylogenetically primitive and are present in single cell organisms presumably as a mechanism for intracellular communication (15). In a chick embryo, $\beta$ receptors have been identified in the myocardium at 2.5 days of incubation and are functional at 5 days of incubation (16). We found that isoproterenol caused a paradoxical increase in vascular resistance mediated through a $\beta$ receptor $(17,18)$. In addition to receptor-mediated response, we found that caffeine, a phosphodiesterase inhibitor, decreased vascular resistance in chick embryos at stage 24 (19).

The increase in vascular resistance in response to hypothermia may be mediated by circulating neurohumoral agents. Wallace (20) found that the embryo produces serotonin as early as stage 10 . In addition, egg yolk contains epinephrine and norepinephrine (21). Although there is no adrenal gland at stages 18 to 24 , a change in environmental temperature may alter the vascular sensitivity to neurohumoral agents that are already present. In the mature duck, resistance vessel's sensitivity to norepinephrine increases 2-fold as the temperature is reduced from 35 to $20^{\circ} \mathrm{C}$ (22). Thus, hypothermia may increase resistance vessel response to a steady state circulating level of a vasoactive drug.

The change in vascular resistance may also be related to the critical closing pressure of the vascular bed. As mean arterial pressure decreases below the critical closing pressure, resistance vessels collapse. This phenomena may explain the resistance change at these stages. Resistance vessel diameter may increase with development: thus, the critical closing pressure will be highest in stage 18 embryos, accounting for the observed changes on rewarming.

These observations may also be important to the understanding of the teratogenic effects of hypothermia. In the chick embryo, a transient reduction in incubation temperature increases the mortality rate and the incidence of cardiac defects in the surviving embryos (1). Although temperature may affect the rate of cell development and cell death, our study defined a profound change in cardiovascular physiology. Numerous indirect investigations have suggested that alteration of cardiac rheology by mechanical interference or drugs leads to a production of cardiac anomalies (23). We speculate that an alteration in blood flow patterns and blood vessel wall stress is an important factor in the pathogenesis of hypothermic-induced cardiac defects.
Acknowledgments. Thanks are due to Dr. Atsuyoshi Takao for encouraging this collaborative project, Sue Kucera for preparation of the manuscript, and Horst Jordan for the graphics.

\section{REFERENCES}

1. deLaCruz M, Campillo-Sainz C, Munz-Armas S 1966 Congenital heart defects in chick embryos subjected to temperature variation. Circ Res 18:257-262

2. Wispé J, Hu N, Clark EB 1983 Response of the chick cardiovascular system to hypothermia. Pediatr Res 17:945-948

3. Hamburger V, Hamilton HL 1951 A series of normal stages in the development of the chick embryo. J Morphol 88:49-92

4. Clark EB, Hu N 1982 Developmental hemodynamic changes in the chick embryo from stage 18 to 27 . Circ Res 51:810-815

5. Finney DJ 1978 Statistical Method in Biological Assay, 3rd ed. MacMillan, New York

6. Cohn AE 1927 Physiological ontogeny. A. Chicken embryos, XIII. The temperature characteristic for the contraction rate of the whole heart. J Gen Physiol 10:369-375

7. Sperelakis N, Lehmkuhl D 1967 Effects of temperature and metabolic poisons on membrane potentials of cultured heart cells. Am J Physiol 213:719-724

8. Girard H 1973 Arterial pressure in the chick embryo. Am J Physiol 224:454460

9. Warbanow W 1971 Contractility of the embryonic chick heart in hypothermiainduced cardiac hyperplasia and hypertrophy. Acta Biol Med Ger 26:859 861

10. Rychter Z 1962 Experimental morphology of the aortic arches and the heart loop in chick embryo. Adv Morphogenesis 2:333-371

11. Thauer $R 1965$ The circulation in hypothermia of nonhibernating animals and men. In: Hamilton W and Dow P (eds) Handbook of Physiology, Section 2 , Vol III. American Physiological Society, Washington DC, pp 1899-1920

12. Pappano AJ 1977 Ontogenic development of autonomic neuroeffector transmission and transmitter reactivity in embryonic and fetal hearts. Pharmacol Rev 29:3-33

13. Higgins D, Pappano A 1981 Development of transmitter secretary mechanisms in adrenergic neurons in the embryonic chick heart ventricle. Dev Biol 87:148-162

14. Romanoff AL 1960 The Avian Embryo: Structure and Functional Development. MacMillan, New York

15. Roth J, Leroith D, Shiloach J, Rosenzweig J, Lesniak M, Havrankova J 1982 The evolutionary origins of hormones, neurotransmitters, and other extracellular chemical messengers. N Engl J Med 306:523-527

16. Lipshultz S, Shanfeld J, Chacko S 1981 Emergence of $\beta$ adrenergic sensitivity in the developing chicken heart. Proc Natl Acad Sci 78:288-292

17. Clark EB, Hu N, Dooley JB 1985 The effect of isoproterenol on cardiovascular function in the stage 24 chick embryo. Teratology 31:41-47

18. Clark EB, Feldman R, Hu N, Lai CC 1984 Paradoxical $\beta$ receptor mediated vasoconstriction in the stage 24 chick embryo. Pediatr Res 18(4)part Il:121A

19. Hawkins J, Hu N, Clark EB 1984 The effect of caffeine on cardiovascular function in the stage 24 chick embryo. Dev Pharmacol Ther 7:334-343

20. Wallace JA 1982 Monoamines in the early chick embryo. Demonstration of serotonin synthesis and regional distribution of serotonin-concentrating cells during morphogenesis. Am J Anat 165:261-274

21. Ignarro $\mathrm{W}$, Shideman FE 1968 Appearance and Concentrations of Catecholamines and their biosynthesis in the embryonic and developing chick. J Pharm Exp Theur 159:38-48

22. Millard RW, Reite OB 1975 Peripheral vascular resistance to norepinephrine at temperatures from $2^{\circ}$ to $40^{\circ} \mathrm{C}$. J Appl Physiol 38:26-32

23. VanPraagh R, Takao R 1980 Etiology and morphogenesis of congenital heart disease. Futura Publishing Company, Mt Kisco, NY 